\title{
Who reports absence of sexual attraction in Britain? Evidence from national probability surveys
}

\author{
Catherine R.H. Aicken ${ }^{a *}$, Catherine H. Mercer ${ }^{\mathrm{a}}$ and Jackie A. Cassell ${ }^{\mathrm{a}, \mathrm{b}}$ \\ ${ }^{a}$ Centre for Sexual Health and HIV Research, Research Department of Infection and Population \\ Health, University College London, London, UK; ${ }^{b}$ Division of Primary Care \& Public Health, \\ Brighton and Sussex Medical School, Falmer, UK
}

(Received March 2011; final version received August 2011)

\begin{abstract}
There is little evidence about the prevalence of absence of sexual attraction, or the characteristics of people reporting this, often labelled asexuals. We examine this using data from two probability surveys of the British general population, conducted in 1990-1991 and 2000-2001. Interviewers administered face-to-face and self-completion questionnaires to people aged $16-44$ years $(N=13,765$ in $1990-1991 ; N=12,110$ in 2000-2001). The proportion that had never experienced sexual attraction was $0.4 \%$ (95\% CI: $0.3-0.5 \%$ ) in $2000-2001$, with no significant variation by gender or age, versus $0.9 \%$ (95\% CI: $0.7-1.1 \%$ ) in $1990-1991 ; p<0.0001$. Among these 79 respondents in 2000-2001, 28 (40.3\% men; 33.9\% women) had had sex, 19 (33.5\% men; $20.9 \%$ women) had child(ren), and 17 (30.1\% men; $19.2 \%$ women) were married. Three-quarters of asexual men and two-thirds of asexual women considered their frequency of sex 'about right', while $24.7 \%$ and $19.4 \%$, respectively, 'always enjoyed having sex'. As well as providing evidence on the distribution of asexuality in Britain, our data suggest that it cannot be assumed that those reporting no sexual attraction are sexually inexperienced or without intimate relationships. We recognise the possibility of social desirability bias given our reliance on self-reported data, but suggest that its effect is not easily predicted regarding absence of sexual attraction.
\end{abstract}

Keywords: asexuality; sexual attraction; survey; sexual behaviour

\section{Introduction}

There is little known about asexual people (Bogaert, 2006; Prause \& Graham, 2007), including whether asexuality is perceived as problematic or associated with ill-health. Asexuality itself is not a well-defined term or phenomenon, and a variety of definitions exist (Hinderliter, 2009), including engagement in no or few sexual behaviours with others (Rothblum \& Brehony, 1993), and asexual self-identification. Definitions based on little or no sexual behaviour may be confounded by, for instance, lack of availability of sexual partners, or non-consensual sexual experiences (Prause \& Graham, 2007). The term 'asexual' as an identity is relatively recent, despite the awareness-raising work of the online Asexuality Visibility and Education Network (AVEN) (Hinderliter, 2009). Though it is defined on AVEN's homepage as 'Asexual: a person who does not experience sexual attraction' (AVEN, 2011), use of the term 'asexual' may be problematic for identifying those who

\footnotetext{
*Corresponding author. Email: c.aicken@ucl.ac.uk 
have not yet 'come out' to an asexual identity (Brotto \& Yule, 2009). A common operational definition would greatly assist research in this area (Brotto \& Yule, 2009). In this article we use absence of sexual attraction to others as a definition but recognise that this definition is contested.

Mixed-methods studies have used convenience sampling to study people identifying as asexual (Brotto, Knudson, Inskip, Rhodes, \& Erskine, 2010; Prause \& Graham, 2007). There are limitations to this sampling method, in terms of the generalisability of findings (Brotto \& Yule, 2009; Prause \& Graham, 2007): it is not possible to know whether those recruited are representative of all asexuals, due to bias in participation. Prause and Graham (2007) conducted a small-scale qualitative study with self-identified asexuals followed by a survey with undergraduate psychology students in a town in the United States, which was also advertised online (without explicitly mentioning asexuality in the advert), thus aiming to reach a mixture of asexuals and sexuals. Brotto et al. (2010) conducted a survey on the AVEN website (AVEN, 2011), comparing this to 'normative data' (i.e. standardised clinical versus non-clinical ranges and cut-offs), followed by a nested qualitative sub-study (participants in the qualitative research were recruited from among the survey participants; for an overview of this type of study design, see Schatz, 2009). Brotto et al.'s study (2010) aimed to focus on asexuals and involved asking respondents to endorse an asexual 'label' to participate, yet $20 \%$ of males and $27 \%$ of females responded that they were not asexual. It was unclear why this was, but it may be that they assumed, given the context, that the question meant something different, for instance, romantic attachments (Brotto et al., 2010). The findings of these studies are largely congruent. Prause and Graham (2007) found that absence of desire for sex with a partner (measured using the Dyadic Sexual Desire subscale of the Sexual Desire Inventor: Spector, Carey, \& Steinberg, 1996) was a particularly strong predictor of asexual self-identity, with lower solitary sexual desire and lower sexual arousability also differentiating asexuals from others with reasonably strong predictability. Brotto et al.'s (2010) findings broadly support this: absence of sexual attraction is a good predictor of self-identifying as asexual, and they also found a lower than normative sexual response among respondents. Meanwhile, a qualitative online survey of AVEN members used open-ended questions to explore the meaning of asexuality in greater depth, with absence of sexual attraction and desire common to the majority of respondents, and a minority reporting sexual activity with others (Scherrer, 2008).

In contrast to data collected from convenience samples, data from population surveys using probability sampling methods enable prevalence estimates to be obtained that can be considered broadly representative of the general population. Bogaert (2004) conducted what appears to be the first study designed to estimate how common absence of sexual attraction is and to compare those reporting this to the rest of the general population. He used data from the first British National Survey of Sexual Attitudes and Lifestyles (Natsal1, Johnson, Wadsworth, Wellings, \& Field, 1994) conducted in 1990-1991. Another study, the National Survey of Family Growth (NSFG, conducted 2002 in the United States) purports to explore asexuality also using national probability survey data (Poston \& Baumle, 2010). It used a question about sexual identity with pre-defined responses, which lacked the response option 'asexual'. In the analysis, it was assumed that asexuals would respond 'something else', instead of heterosexual, homosexual, or bisexual (Poston \& Baumle, 2010). However, in order to pick responses that best fit their experience, asexuals may in fact categorise romantic relationships in which they do not necessarily feel sexual attraction, as hetero-, homo-, or bisexual, and not 'something else' (Brotto et al., 2010). Similarly, a question in the NSFG about sexual attraction had no response option to indicate no attraction, and in the analysis of this study it was assumed that asexuals would respond 
'not sure' (Poston \& Baumle, 2010). The measure may therefore be over-inclusive, as respondents who do experience sexual attraction, but are not sure about their sexual preference, may also select 'not sure', as the authors recognise, or may not respond at all (Poston \& Baumle, 2010). Thus, until now, Bogaert's (2004) study has been the only one to measure reported absence of sexual attraction in the general population, as far as we are aware. The question it relies upon, and which has been used in the more recent Natsal2 survey in 2000-2001, is an adaptation of the Kinsey scale (Kinsey, Pomeroy, \& Martin, 1948), which rates sexual attraction and experience, from entirely homosexual through to bisexual to entirely heterosexual, only sometimes referred to as including an asexual category. In the Natsal surveys, a five-point scale of sexual attraction is used, ranging from only to the opposite gender to solely to the same gender, with a sixth option 'I have never been sexually attracted to anyone at all' (Johnson et al., 1994) to make the question more widely applicable.

Bogaert's (2004) work based on Natsal-1 is often cited in the small literature on asexuality. As such, later studies refer to Bogaert's use of this definition in such a way that it seems that the formulation of the question in the Natsal survey, which serves a variety of medical and social purposes, may have driven the operational definition used in subsequent debate. Despite the finding that lack of sexual attraction to others has a high sensitivity yet poor specificity relative to self-identifying as asexual (Prause \& Graham, 2007), ${ }^{1}$ it is reassuring that absence of sexual attraction to others does seem central to a definition of asexuality based on others' findings (Brotto et al., 2010; Prause \& Graham, 2007).

In this article we estimate the prevalence of absence of sexual attraction in Britain using data from the most recent Natsal study (Natsal-2, 2000-2001). We examine change in this prevalence since 1990-1991, using the earlier Natsal-1 data, which Bogaert used (2004). In the more recent data from Natsal-2, we compare those reporting no sexual attraction with other respondents, in terms of socio demographics, sexual behaviours, and attitudes.

\section{Methods}

\section{Population and study design}

The National Surveys of Sexual Attitudes and Lifestyles (Natsal) are stratified probability sample surveys of the general population resident in Britain undertaken in 1990-1991 and 2000-2001, and are to date the largest surveys of sexual behaviour anywhere in the world. Details of the methodology and question wording are published elsewhere (Economic and Social Data Service, 2011; Erens et al., 2001; Johnson et al., 1994; Johnson, Mercer et al., 2001). Briefly, in 1990-1991 Natsal-1 interviewed 13,765 people aged 16-59 years (Johnson et al., 1994; Wellings, Field, Johnson, Wadsworth, \& Bradshaw, 1994), while a decade later in 2000-2001 Natsal-2 interviewed 12,110 people aged 16-44 years (Johnson, Copas et al., 2001; Johnson, Mercer et al., 2001; Wellings et al., 2001), reflecting the concentration of sexually transmitted infections (STI)/HIV risk behaviours in those aged under 45 years observed in Natsal-1. Natsal-1 and Natsal-2 achieved similar response rates, $66.8 \%$ and $65.4 \%$, respectively, which are in line with other major British surveys (Lynn \& Clarke, 2002).

Respondents were interviewed at home with a questionnaire, which involved a face-toface interview carried out by highly trained interviewers and then a self-completion module, using pen-and-paper interviewing in Natsal-1 and computer-assisted self-interviewing in Natsal-2. Towards the end of the face-to-face interview in both Natsal-1 and Natsal-2, all respondents were given a show card with a question and coded response options shown below, and were given the following instruction: 
Now please read this card carefully as it is important that you understand it and are as honest as you can be in your answer. When you've finished reading, tell me which letter represents your answer.

I have felt sexually attracted ...

(1) (K) Only to females, never to males

(2) (C) More often to females, and at least once to a male

(3) (F) About equally often to females and to males

(4) (L) More often to males, and at least once to a female

(5) (D) Only ever to males, never to females

(6) (N) I have never felt sexually attracted to anyone at all

From the responses to this question, we created a binary variable to identify respondents who reported that they had never felt sexually attracted to anyone at all, referred hereon for brevity as an absence of sexual attraction.

Natsal surveys use a broad definition of sexual intercourse, and respondents are asked to include as sexual partners anyone with whom they have had oral, vaginal, and/or anal sex.

\section{Statistical analyses}

Analyses were performed using STATA 9.2 to account for stratification, clustering, and weighting of the samples (Stata Corporation, 2006). Each survey's data were weighted to correct for unequal selection probabilities and to match the corresponding age/sex population profile (Erens et al., 2001; Johnson, Mercer et al., 2001).

We compared the percentages reporting an absence of sexual attraction in Natsal-1 and Natsal-2 by gender and then used logistic regression to obtain age-adjusted odds ratios for reporting this in Natsal-2 relative to Natsal-1, which we treat as the reference category.

We then compared the reporting of selected socio demographics, sexual behaviours, and attitudes by respondents in Natsal-2 according to whether or not they reported an absence of sexual attraction. We used the chi-square statistic to determine whether differences between categorical variables were statistically significant, and the $t$-test statistic to determine whether differences between normally distributed continuous variables were statistically significant, which we considered to be $p<0.05$ in all analyses.

\section{Ethical approval}

The Natsal-2 study was approved by the University College Hospital and North Thames Multi-Centre Research Ethics Committee and all the Local Research Ethics Committees in Britain.

\section{Results}

\section{Prevalence of asexuality}

From the most recent Natsal data (Natsal-2, 2000-2001) it is estimated that among those aged 16-44 years, $0.3 \%$ (95\% CI: $0.2-0.5 \%$, weighted/unweighted $N: 20 / 26)$ of men 
and $0.5 \%$ (95\% CI: $0.4-0.7 \%$, weighted/unweighted $N: 30 / 53)$ of women had never been sexually attracted to anyone (no statistically significant gender difference; thus the overall prevalence is estimated to be $0.4 \%$ [95\% CI: $0.3-0.5 \%]$ ). This prevalence estimate is significantly smaller than estimated from Natsal-1 in 1990-1991 among those aged 16-44 years: $0.8 \%$ (95\% CI: $0.6-1.1 \%$, weighted/unweighted $N: 73 / 72)$ among men; $1.0 \%$ (95\% CI: $0.8-1.3 \%$, weighted/unweighted $N$ : $95 / 128)$ among women; $0.9 \%$ (95\% CI: $0.7-1.1 \%$, weighted/unweighted $N: 168 / 200)$ was the overall prevalence in 1990 . The corresponding age-adjusted odds ratios (ORs) are 0.44 (95\% CI: $0.25-0.80)$ and 0.52 (95\% CI: $0.35-0.78)$, among men and women, respectively. There is no statistically significant gender difference in the magnitude of this decline between 1990 and 2000.

In 2000, men who experienced sexual attraction to others had a mean age of 28.5 years (95\% CI: $23.7-33.3$ years), while those with an absence of sexual attraction had a mean age of 30.6 years (95\% CI: $30.3-30.8$ years; $t$-test statistic -0.86 ; $p$-value for difference: 0.392 ). The corresponding mean ages for women were: 30.7 years (95\% CI: 30.4-30.9 years) among those who experienced sexual attraction; and among those with an absence of sexual attraction, 29.4 years (95\% CI: $26.3-32.5$ years; $t$-test statistic -0.80 ; $p$-value for difference: 0.426 ).

\section{Sexual experience, sexual partnerships, and relationships of asexuals in 2000-2001}

Among those with an absence of sexual attraction in 2000, two-thirds of women $(66.1 \%)$ and a slightly smaller proportion of men (59.7\%) reported never having had a sexual partner of either gender, in contrast to $4.9 \%$ of women and $6.0 \%$ of men who had experienced sexual attraction (Table 1). Among both men and women, we found no statistical evidence for a difference in the proportion ever having had a same-sex partner, between those reporting no sexual attraction, and those reporting sexual attraction to either or both genders. Among those with an absence of sexual attraction but who did report sexual partner(s), the median number of partners ever was 1 (with an upper quartile of 5 partners among men and 3 partners among women). The data for the more recent time-frames (the last five years and last year) are similar: the majority of respondents with an absence of sexual attraction had not had any sexual partners, but a non-negligible minority had at least one sexual partner (in the last five years and the last year, respectively: $33.3 \%, 27.3 \%$ of men and $26.7 \%$, $26.6 \%$ of women).

\section{Religion and ethnicity}

We also found some evidence for differences by ethnicity. Absence of sexual attraction was more commonly reported among respondents of Indian and Pakistani ethnic origin, who made up $12.2 \%$ men and $23.2 \%$ of women without sexual attraction (in contrast to $2.7 \%$ and $2.8 \%$ of those who experienced sexual attraction). The differences seemed to be more pronounced among women, and among Pakistani respondents compared to Indian respondents. While we found no evidence for a difference in the proportions who belonged to a religion (compared to having no religion), there were significant differences between religious affiliations, among women. Of note, a larger proportion of respondents reporting an absence of sexual attraction were Muslim (11.6\% versus $2.1 \%$ among men; $19.2 \%$ versus $2.1 \%$ among women), while a smaller proportion reported a Christian-based faith $(27.5 \%$ versus $35.7 \%$ among men; $33.7 \%$ versus $45.5 \%$ among women). 


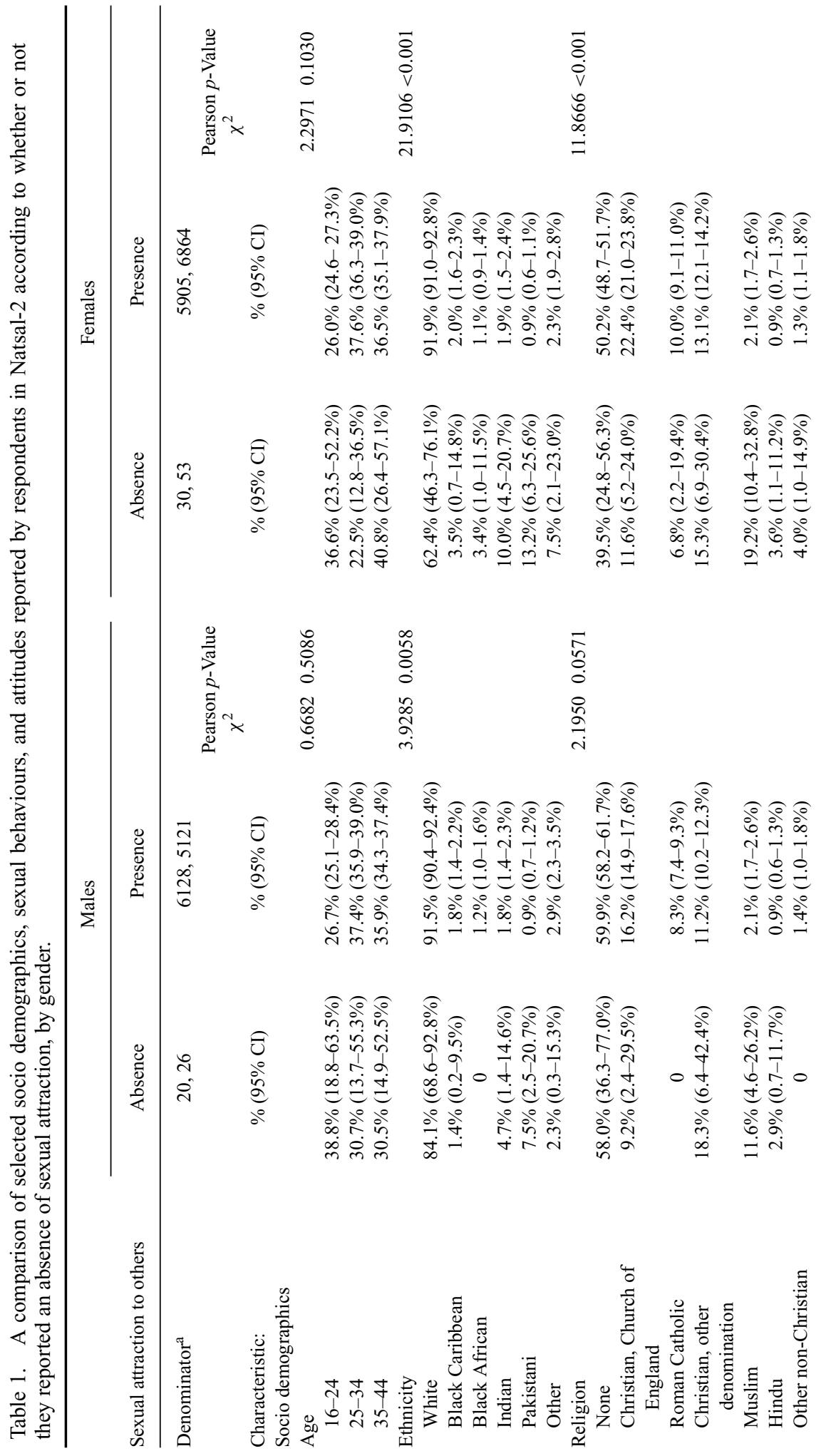


$\overrightarrow{8}$
$\dot{0}$
$v$
守
m.
?

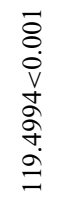

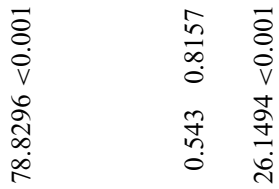

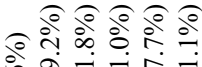

bั $\stackrel{i}{\sim} \vec{\sim}$

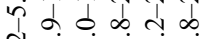

ป

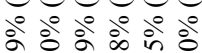

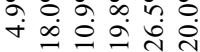

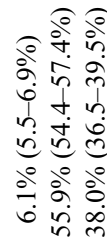

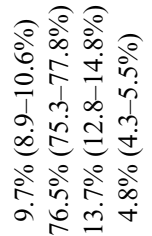

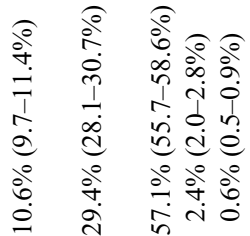

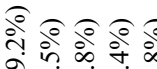

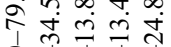

$0.4 \frac{1}{1}$

roesd

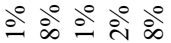

ठ̊.

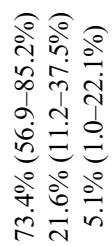

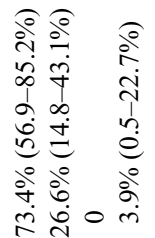
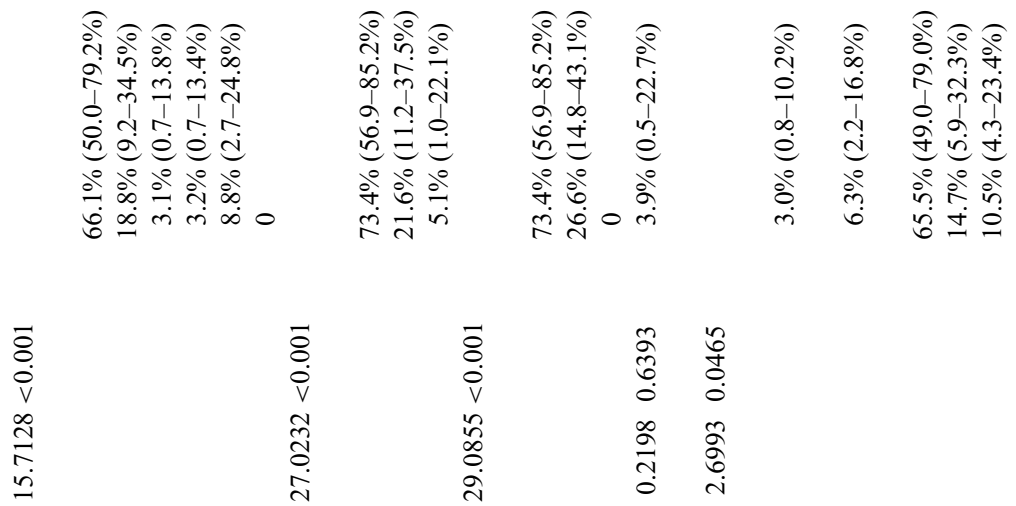

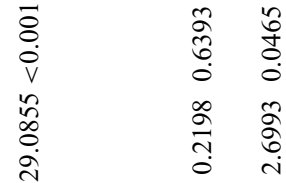

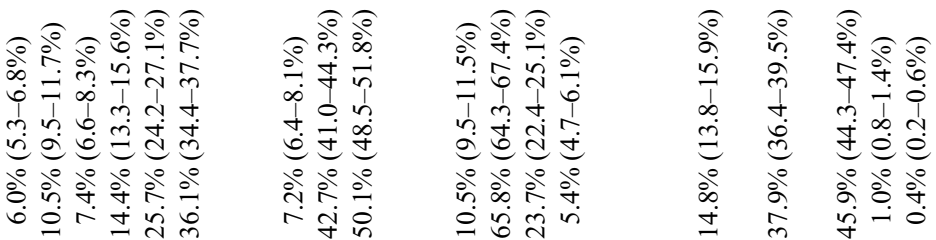

\begin{tabular}{|c|c|c|c|c|c|}
\hline 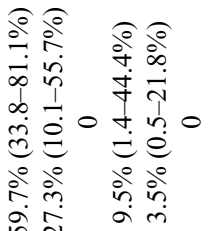 & 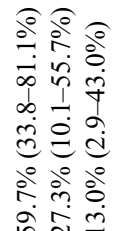 & 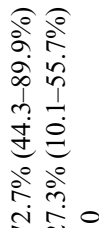 & 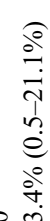 & 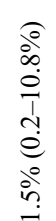 & 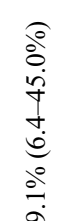 \\
\hline
\end{tabular}

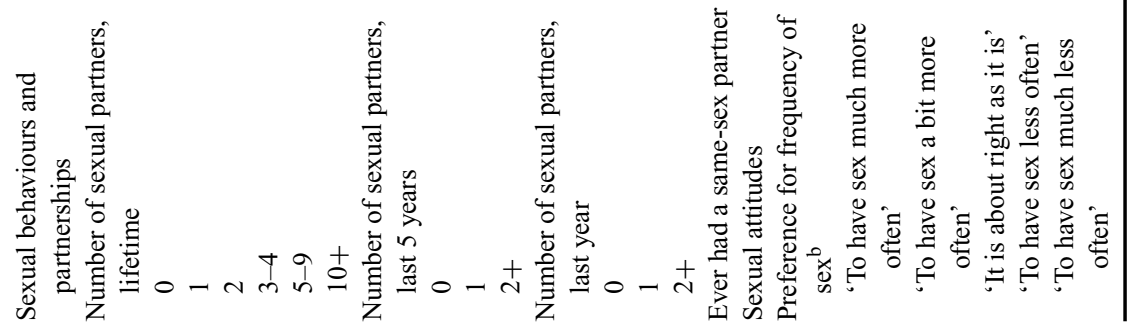




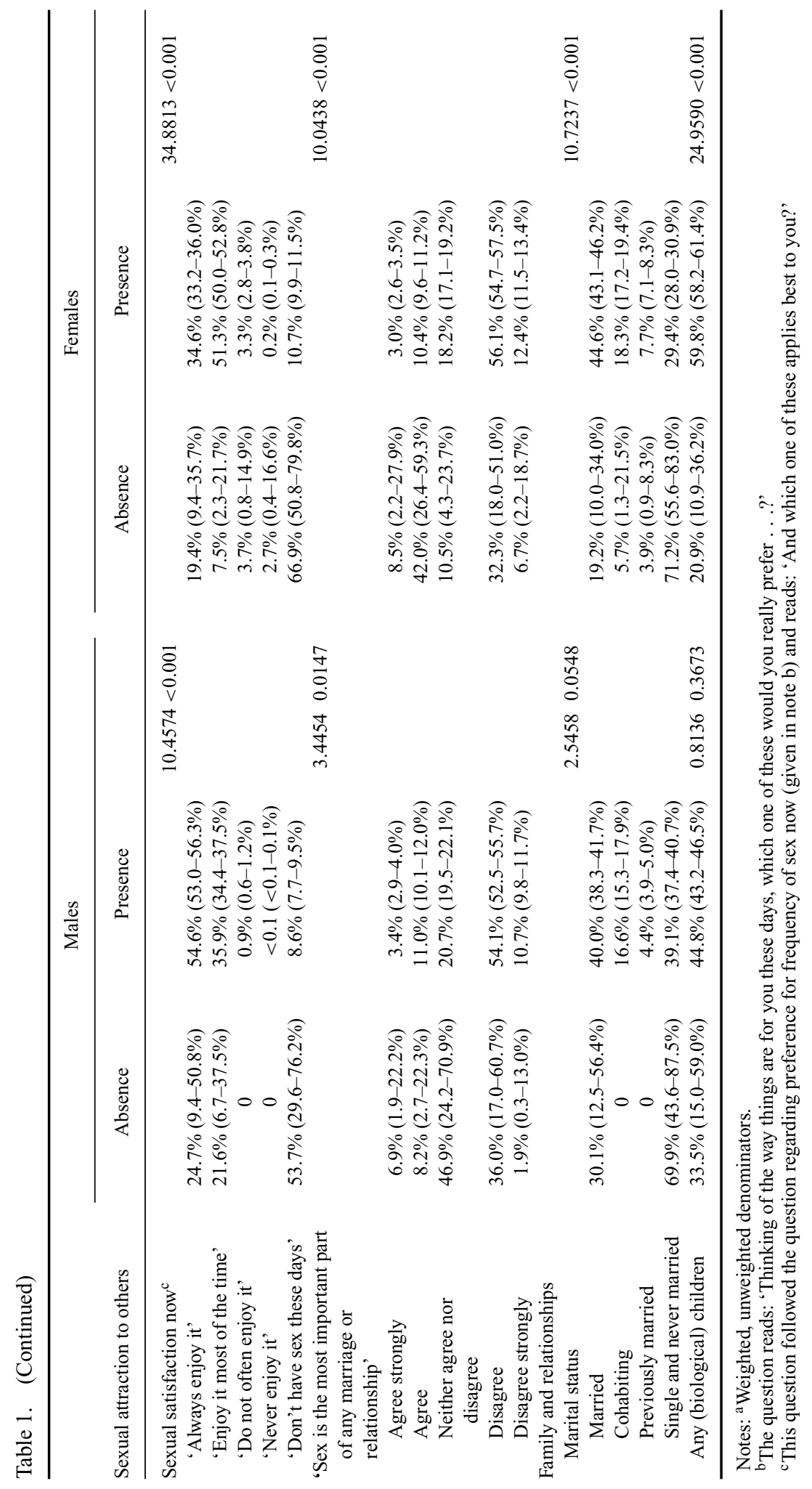




\section{Current sexual lifestyle and attitudes}

More respondents who reported an absence of sexual attraction (69.9\% men, 71.2\% women) described their marital status as 'single, never married', than respondents reporting sexual attraction to others (39.1\% men, $29.4 \%$ women). Nevertheless, almost a third of men and a quarter of women with an absence of sexual attraction said that they were married or cohabiting with a partner. Among men this difference was of borderline statistical significance but among women it was highly significant $(p=0.0548, p<0.001$ respectively; chi-squared statistics and percentages are presented in Table 1). One in three men and one in five women with an absence of sexual attraction had children, in contrast to almost half of men and almost two-thirds of women with sexual attraction. Among women (but not men) this reached statistical significance.

There were also differences in the sexual attitudes examined by the Natsal-2 survey, described below (also see Table 1). More people with an absence of sexual attraction considered their frequency of sex to be 'about right as it is' in contrast to the rest of the population: $76.0 \%$ versus $45.9 \%$, respectively, among men; $65.5 \%$ versus $57.1 \%$, respectively, among women. Only one in five men and one in 10 women reported that they would like sex either 'much more often' or 'a bit more often' among those with an absence of sexual attraction, compared to more than half of men and one in three women who did experience sexual attraction. Most of those with an absence of sexual attraction indicated that they 'don't have sex these days' (53.7\% men, 66.9\% women), but among the 23 asexuals who had had sex in the last year, all seven men and 10 of the women reported that they either 'always enjoy it' or 'enjoy it most of the time'.

Natsal-2 asked respondents the extent to which they agreed with the statement 'sex is the most important part of any marriage or relationship'. Among those with an absence of sexual attraction, $37.9 \%$ men and $39.0 \%$ women disagreed or disagreed strongly, in contrast to $64.8 \%$ and $68.5 \%$ of other respondents. Surprisingly, more than half of women without sexual attraction agreed or agreed strongly to the statement.

\section{Discussion}

\section{Main findings}

This analysis of the most recent (at the time of writing) Natsal survey data suggests that a small proportion of people aged 16-44 years in Britain experience an absence of sexual attraction and that the prevalence does not vary with gender or age. Data from the two Natsal surveys suggest that the proportion of all respondents with an absence of sexual attraction declined between 1990 and 2000, yet the magnitude of this change has been similar for men and women. Respondents who reported no sexual attraction were, perhaps unsurprisingly, more likely to report never having had sexual intercourse, to be unmarried, or cohabiting with a partner, and to have no children. In each case this was far from universal, however, with over a third of those reporting no sexual attraction reporting at least one sexual partner, around a quarter married or cohabiting, and a similar proportion reporting that they have (biological) children.

\section{Relationship to other studies}

We restricted the age group for analysis to those aged 16-44 years, which allowed comparability between Natsal surveys. This may account for differences between our findings and those of Bogaert (2004), particularly the association he found with age. We found no 
such association, consistent with an analysis of the US NSFG, a probability survey with a similarly aged target population: 15-44 years (Poston \& Baumle, 2010). Differences to the findings of other studies may be due to differences in study and sample design, or due to genuine differences in the populations from which the samples were drawn.

Absence of sexual attraction differed by religious affiliation, particularly amongst women: our data suggest that absence of sexual attraction is more common among Muslims and less common among Church of England Christians. Bogaert (2004) found that more frequent attendance at religious services was associated with absence of sexual attraction, and perhaps similar explanations apply, as many Church of England Christians do not attend church regularly. ${ }^{2}$ Qualitative research with members of AVEN (a self-selected group of those identifying as asexual), suggested atheism was common among members (Brotto et al., 2010). However, we found that very similar proportions of men with and without sexual attraction had no religion $(59.9 \%, 58.0 \%)$, while our data suggest a possible difference among women, in the opposite direction (50.2\% of those with versus $39.5 \%$ without sexual attraction). It is of course possible that those who experience an absence of sexual attraction and are religious may be more likely to find support and acceptance for this through their religion, while for atheists, networks such as AVEN, and the adoption of an asexual identity, may have greater appeal.

Cultural and religious norms could relate to differences we observed between ethnic groups: the higher prevalence of absence of sexual attraction reported by respondents of Pakistani and Indian ethnicity (and particularly women from these groups), relative to white respondents. This might, for instance, reflect a perceived norm of universal sexual attraction more common in the white population. Differences in sexual behaviour by ethnicity and gender in the United Kingdom (Fenton et al., 2005; Saxena et al., 2006; Sinha, Curtis, Jayakody, Viner, \& Roberts, 2007) also suggest differing sexual norms, perhaps related to 'true' differences in the proportion experiencing sexual attraction. It must also be noted that although Natsal-2 over-sampled the four main ethnic minority groups in the United Kingdom (which was accounted for in the weighting of these data), the numbers reporting absence of sexual attraction within each ethnicity category were very small. Other differences, for instance socio-economic differences (Sinha et al., 2007), may confound associations with ethnicity. The complexity of this, and the interplay among gender, culture, religion, and the experience of sexual attraction, are beyond the scope of this article, but merit further investigation.

The sizeable proportion of respondents without sexual attraction who reported sexual activity and current sexual partnerships is consistent with the diversity of asexual experience frequently described in the literature (AVEN, 2011; Brotto \& Yule, 2009; Hinderliter, 2009). Though due to study design differences it would be inappropriate to make direct comparisons, Brotto et al. (2010) found that many of their sample of AVEN members were in relationships, some of them sexual relationships. Interestingly, the analysis of US NSFG data found no difference between females reporting sexual attraction and those responding 'not sure', in the odds of ever having been married, but men who responded 'not sure' were less likely ever to have been married (there was no difference among men or women in the odds of ever having cohabited).

\section{Possible explanations and limitations of the study in choosing between them}

Social desirability bias is particularly interesting in relation to absence of sexual desire, as there are compelling arguments for its operation in either direction (or differently among different individuals or groups): resulting in either over- or under-reporting of sexual 
attraction. For instance, the reduction in prevalence between surveys may reflect a genuine change over time in the experience of sexual attraction in the population. Alternatively, there may have been changes affecting the reporting of sexual attraction. The social desirability of reporting an absence of sexual attraction may have decreased, and/or the social undesirability of reporting it may have increased. While widely recognised as 'normal', dependent on an individual's circumstances and values, it could be seen as undesirable to report either sexual attraction or its absence. Though changes in societal norms could increase pressure to report some sexual attraction, it may be argued that an absence of sexual attraction has, until comparatively recently, been viewed as a virtue.

The Natsal studies, and indeed all surveys, gather self-reported data and reporting bias (including social desirability bias) can be reduced but cannot be entirely eliminated (Mercer, McManus, \& Erens, 2010). Confidentiality (assured in both Natsal surveys) and administration by computer-assisted personal interview (CAPI) may increase the reporting of socially undesirable or stigmatised experiences (Johnson, Copas et al., 2001). In this respect it may be relevant that Natsal-1 was administered as a pen-and-paper interview while Natsal-2 was administered in a computerised format (CAPI). However, in both surveys, the question was asked in the section of the survey administered by the interviewer, so the effect of the different mode of recording may be minimal. It has also been noted that those who experience no sexual attraction might be less likely to participate in a survey of this type (i.e. participation bias), hence it is possible that we underestimate the true prevalence of absence of sexual attraction. Sexual attraction can be measured in physiological ways, avoiding the problems of using self-reported data; however, recruitment to these types of studies may be skewed towards people with more sexual experience (Bogaert, 2006), and this effect would conceivably be greater than for survey studies such as Natsal.

The data in Natsal were broadly congruent with the findings of other studies (Brotto et al., 2010; Prause \& Graham, 2007), yet the question used in the Natsal surveys asks about never having experienced sexual attraction to another person and so excludes those who experience very little sexual attraction. It also 'requires asexuality to be lifelong and absolute' rather than more fluid (Hinderliter, 2009), so would underestimate the proportion of people experiencing no recent sexual attraction. Further, it may be difficult for someone who has not experienced sexual attraction to know what it feels like, particularly when they may feel other forms of attraction, e.g. romantic attraction (Hinderliter, 2009). Differences in understanding of questions may apply to all in survey and interview research, yet can be reduced through cognitive pre-testing (although in the testing process, it may be difficult to recruit sufficient numbers of people with rare experiences - Aicken et al., 2013). We note criticisms of the measure we used (Hinderliter, 2009; Prause \& Graham, 2007), which appeared in the literature in response to Bogaert's (2004) use of the Natsal-1 data (Bogaert's analysis was published after Natsal-2 had already been conducted), notably the absence of a question on sexual identity. Furthermore, the measure we use is not sufficiently sensitive to distinguish between asexuals who experience romantic attraction and those who do not (romantic and aromantic asexuals). Among those not experiencing sexual attraction, it is likely that factors such as marital status, sexual activity, having biological children - and indeed attitudes to sex and marriage - may vary between these sub-populations, with romantic asexuals perhaps constituting many of those who report marriage, sexual activity, and having children, yet no sexual attraction. Due to the Natsal definitions of sexual intercourse and sexual partners (outlined in the Methods), we also cannot distinguish between sexual partners with whom only oral sex, but not vaginal or anal sex, occurred, and it is possible that for some asexuals the former may be acceptable but not the latter. More broadly, we note that where attempts are made to make survey 
questions suitable for the measurement of sexualities (e.g. through the work of the Sexual Minority Assessment Research Team, 2009), asexual identity and absence of sexual attraction are often overlooked. One unfortunate result of this is that it is difficult to tell whether there are real differences in the prevalence and distribution of absence of sexual attraction between the United Kingdom and United States, because of the lack of an appropriate response category in the NSFG (Poston \& Baumle, 2010). Indeed, global concerns over the human rights of gay, lesbian, and bisexual people may appear to overshadow any discrimination against asexuals, further contributing to the lower visibility of asexuals in sexuality research.

Of course, with our methodology we are not equipped to explore the meaning that an asexual identity or absence of sexual attraction has to a person's life, though this is an important area of study (Brotto et al., 2010). Our quantitative methodology does, however, allow us to estimate how common absence of sexual attraction is in the general population and to explore the demographic and other characteristics of those who report it. There is a general problem with quantitative research on rare population groups that lack a sampling frame. For probability sampling, very large sample sizes are required in order to obtain representative samples of the rare group, yet studies seeking to target their recruit to members of this rare group, though they may attain greater numbers, cannot determine whether the sample is representative (increasing the sample size does nothing to remove the effect of bias if the sampling strategy is not representative). Kinsey's data are considered 'highly selective and unrepresentative' (Hart \& Wellings, 2002) for the reason that he sought out less common behaviours. A further problem with the use of population surveys is that they are often designed to serve many purposes, as Natsal is, and response options must reflect a broad range of experiences. In the sensitive area of sexual behaviour, there are also concerns about achieving reliable results, minimising non-response and minimising the risk of the respondent terminating the interview (Aicken et al., 2013). Response options likely to be selected by a very small minority of respondents (e.g. intersex) may be excluded, particularly where the numbers achievable are unlikely to give sufficient statistical power to be able to describe the group in any meaningful way.

\section{Meaning of this study}

Much research around sexuality focuses on sexual behaviour. Perhaps this is unsurprising given the prevailing interest, in public health research, in prevention of infection (Department of Health, 2001), and early, and more generally, unintended pregnancy (Department for Children Schools and Families \& Department of Health, 2010; Social Exclusion Unit, 1999) with an explicit interest in those who have sex young, with many partners, and/or unsafely. This focus may overlook asexuality, assuming it to be associated with absence of risk of disease. Our findings confirm that it cannot be assumed that those not experiencing sexual attraction are not sexually active - indeed, among those who are sexually active, enjoyment of sex is common (and more common than non-enjoyment). Similar findings were reported by studies of asexuals: although the large majority in Brotto et al.'s study (2010) preferred no or very little sex (less than twice a year) and $27 \%$ had never had sexual intercourse, a small proportion (4.8\% men, $2.1 \%$ women) said their ideal frequency of sex was more than once a week. Satisfaction with the frequency of sex (not too much or too little) was actually higher among those reporting no sexual attraction, and some would prefer more sex. This may reflect motivations for having sex, which are diverse (see, for example: Cooper, Shapiro, \& Powers, 1998; Ott, Millstein, Ofner, \& HalpernFelsher, 2006) and are not limited to sexual attraction. However, a quarter of women with an absence of sexual attraction would prefer to have sex less or much less often, contrasting 
with a much smaller proportion of men and other women. While our data suggest that sexual activity among those without sexual attraction does not automatically indicate coercion, ability to express this preference for less sex may be a particular issue for some women with an absence of sexual attraction, which could be addressed through individual counselling, or pre-emptively through improved sex education and sexual health promotion.

We were unable to explore asexual identity, yet the findings of studies with targeted recruitment of asexuals suggest that an asexual identity is not, per se, problematic. Brotto et al. (2010) found the majority of asexuals expressed no distress with their asexual identity, and Prause and Graham (2007) also found no more sexual distress among asexuals than sexuals. The latter remark that any personal distress may be exacerbated by a psychiatric diagnosis and state that 'it remains to be determined to what extent asexuality is problematic in the absence of individual, personal distress' (Prause \& Graham, 2007, p. 353), and we would suggest that the same may apply to absence of sexual attraction.

In our analysis, we look at the demographic descriptors of those reporting absence of sexual attraction, rather than health conditions or disability. (Natsal-3, for which fieldwork was completed in 2012, includes a larger section of health-related questions to find out how this affects sexual function. For a comprehensive discussion around the supposed asexuality of people with disabilities, see Milligan \& Neufeldt, 2001.) The norms around sexual behaviour inadvertently created by population studies (including Natsal) may be problematic, potentially reinforcing feelings of inadequacy (Hart \& Wellings, 2002), thus, perhaps, distress. In contrast to bisexuality and homosexuality, asexuality may have escaped the legal attention common to other marginalised sexualities 'perhaps in part because it is lack of behavior or desire' (Scherrer, 2008, p. 637). Health and disability have been studied in relation to asexuality (variously defined - Bogaert, 2004; Poston \& Baumle, 2010) and it is possible that pre-existing assumptions (Milligan \& Neufeldt, 2001) have driven the study of asexuality in the direction of health and disability, and consequently its medicalisation. Yet, while unusual, we see no evidence that absence of sexual attraction is problematic; and as others note (Bogaert, 2006; Brotto et al., 2010; Prause \& Graham, 2007), distress concerning this may in itself be problematic. Our findings suggest individuals reporting no sexual attraction may or may not be sexually active or in relationships, yet are often satisfied with their frequency of sex. Health and psychology professionals should therefore neither assume that absence of sexual desire is problematic for the individuals who experience it, nor that it implies an absence of sexual experience and romantic relationships, hence these individuals may still have sexual health or relationship counselling needs.

\section{Acknowledgements}

The Natsal-1 study was supported by the Wellcome Trust, while the Natsal-2 study was supported by a grant from the Medical Research Council with funds from the Department of Health, the Scottish Executive and the National Assembly for Wales. The views expressed in this article are those of the authors and do not necessarily reflect the views of the funding bodies.

\section{Notes}

1. That is, many of those who self-identify as asexual say that they lack sexual attraction to others, but the reverse is less true.

2. While around 4 in 10 in England regard themselves as belonging to the Church of England, just 1.7 million attend a church service each month (Church of England, 2011) of England's population of over 49 million (based on data from the 2001 census, retrieved from Neighbourhood Statistics, 2011). 


\section{Notes on contributors}

Catherine R.H. Aicken is an MPhil/PhD student in Public Health. She has worked on a wide range of multidisciplinary studies concerning sexual, reproductive, and other areas of health and health promotion; in recent years she has been focusing on sexual health services research.

Catherine H. Mercer $(\mathrm{PhD})$ is a Senior Lecturer in Sexual Health and the lead statistician for the British National Surveys of Sexual Attitudes and Lifestyles (Natsal-2, Natsal-3). Her research interests include the measurement of sexual behaviour through quantitative surveys; and the use of survey and routinely collected health service data to assist the evidence-based planning of sexual health services.

Professor Jackie A. Cassell is based at Brighton and Sussex Medical School, UK. She has published widely in the field of sexually transmitted infections, focussing on primary care and the impact of delayed care on disease transmission. She currently leads research on partner notification in primary care and other community settings, working closely with the Health Protection Agency, University College London, and a GUM clinic in Eastbourne, where she holds honorary posts. Jackie also leads the Wellcome Trust funded programme, 'The ergonomics of electronic patient records'.

\section{References}

Aicken, C.R.H., Gray, M., Clifton, S., Tanton, C., Field, N., Sonnenberg, P., . . Mercer, C.H. (2013). Improving questions on sexual partnerships: Lessons learned from cognitive interviews for Britain's third National Survey of Sexual Attitudes and Lifestyles ('Natsal 3'). Archives of Sexual Behavior, 42, 173-185. doi: 10.1007/s10508-012-9962-2

Asexuality Visibility and Education Network (AVEN). (2011). Asexuality visibility and education network website. Retrieved from www.asexuality.org

Bogaert, A.F. (2004). Asexuality: Prevalence and associated factors in a national probability sample. Journal of Sex Research, 41, 279-287. doi:10.1080/00224490409552235

Bogaert, A.F. (2006). Towards a conceptual understanding of asexuality. Review of General Psychology, 10, 241-250. doi: 10.1037/1089-2680.10.3.241

Brotto, L.A., Knudson, G., Inskip, J., Rhodes, K., \& Erskine, Y. (2010). Asexuality: A mixedmethods approach. Archives of Sexual Behavior, 39, 599-618. doi:10.1007/s10508-008-9434-x

Brotto, L.A., \& Yule, M.A. (2009). Reply to Hinderliter (2009). Archives of Sexual Behavior, 38, 622-623. doi:10.1007/s10508-009-9514-6

Church of England. (2011). Facts and stats. Retrieved from http://www.churchofengland.org/aboutus/facts-stats.aspx

Cooper, M.L., Shapiro, C.M., \& Powers, A.M. (1998). Motivations for sex and risky sexual behavior among adolescents and young adults: A functional perspective. Journal of Personality and Social Psychology, 75, 1528-1558. Retrieved from http://psycnet.apa.org/index.cfm?fa=browsePA volumes\&jcode $=\mathrm{psp}$

Department for Children Schools and Families \& Department of Health. (2010). Teenage pregnancy strategy: Beyond 2010. Nottingham: Department for Children Schools and Families Publications.

Department of Health. (2001). The national strategy for sexual health and HIV. London: Department of Health.

Economic and Social Data Service. (2011). National survey of sexual attitudes and lifestyles list of datasets. Retrieved from http://www.esds.ac.uk/findingData/natsalTitles.asp

Erens, B., McManus, S., Field, J., Korovessis, C., Johnson, A.M., \& Fenton, K.A. (2001). National survey of sexual attitudes and lifestyles II: Technical report. London: National Centre for Social Research.

Fenton, K.A., Mercer, C.H., McManus, S., Erens, B., Wellings, K., Macdowall, W., ... Johnson, A.M. (2005). Ethnic variations in sexual behaviour in Great Britain and risk of sexually transmitted infections: A probability survey. Lancet, 365, 1246-1255. doi: 10.1016/S0140-6736(05)74813-3

Hart, G., \& Wellings, K. (2002). Sexual behaviour and its medicalisation: In sickness and in health. British Medical Journal, 324, 896-900. Retrieved from www.bmj.com

Hinderliter, A.C. (2009). Methodological issues for studying asexuality. Archives of Sexual Behavior, 38, 619-621. doi:10.1007/s10508-009-9502-x 
Johnson, A.M., Copas, A.J., Erens, B., Mandalia, S., Fenton, K., Korovessis, C., . . Field, J. (2001). Effect of computer-assisted self-interviews on reporting of sexual HIV risk behaviours in a general population sample: A methodological experiment. AIDS, 15, 111-115. Retrieved from http://journals.lww.com/aidsonline/pages/default.aspx

Johnson, A.M., Mercer, C.H., Erens, B., Copas, A.J., McManus, S., Wellings, K., . . Field, J. (2001). Sexual behaviour in Britain: Partnerships, practices, and HIV risk behaviours. Lancet, 358, 1835-1842. doi:10.1016/S0140-67360106883-0

Johnson, A.M., Wadsworth, J., Wellings, K., \& Field, J. (1994). Sexual attitudes and lifestyles. Oxford: Blackwell Scientific Publications.

Kinsey, A.C., Pomeroy, W.B., \& Martin, C.E. (1948). Sexual behavior in the human male. Philadelphia, PA: W.B. Saunders.

Lynn, P., \& Clarke, P. (2002). Separating refusal bias and non-contact bias: Evidence from UK national surveys. Journal of the Royal Statistical Society Series D - The Statistician, 51, 319-333. Retrieved from http://onlinelibrary.wiley.com/journal/10.1111/\%28ISSN\%2914679884

Mercer, C.H., McManus, S., \& Erens, B. (2010). Measuring sexual behaviour. In M. Bulmer, J. Gibbs, \& L. Hyman (Eds.), Social measurement through social surveys: An applied approach (pp. 127-146). Farnham: Ashgate.

Milligan, M.S., \& Neufeldt, A.H. (2001). The myth of asexuality: A survey of social and empirical evidence. Sexuality and Disability, 19, 91-109. doi:10.1023/A:1010621705591

Neighbourhood Statistics. (2011). England and Wales 2001 census data. Retrieved from http://www. neighbourhood.statistics.gov.uk/

Ott, M.A., Millstein, S.G., Ofner, S., \& Halpern-Felsher, B.L. (2006). Greater expectations: Adolescents' positive motivations for sex. Perspectives on Sexual and Reproductive Health, 38 , 84-89. doi: $10.1363 / 3808406$

Poston, D.L., Jr., \& Baumle, A.K. (2010). Patterns of asexuality in the United States. Demographic Research, 23, 509-530. doi:10.4054/DemRes.2010.23.18

Prause, N., \& Graham, C.A. (2007). Asexuality: Classification and characterization. Archives of Sexual Behavior, 36, 341-356. doi:10.1007/s10508-006-9142-3

Rothblum, E.D., \& Brehony, K.A. (1993). Boston marriages: Romantic but asexual relationships among contemporary lesbians. Amherst: University of Massachusetts Press.

Saxena, S., Copas, A.J., Mercer, C.H., Johnson, A.M., Fenton, K.A., Erens, B., ... Wellings, K. (2006). Ethnic variations in sexual activity and contraceptive use: National cross-sectional survey. Contraception, 74, 224-233. doi:10.1016/j.contraception.2006.03.025

Schatz, E. (2009). Nesting semi-structured interviews in surveys or censuses: More than the sum of the parts [Working paper]. Boulder: Institute of Behavioral Science, University of Colorado at Boulder. Retrieved from http://www.colorado.edu/ibs/pubs/pop/pop2009-0008.pdf

Scherrer, K.S. (2008). Coming to an asexual identity: Negotiating identity, negotiating desire. Sexualities, 11, 621-641. doi:10.1177/1363460708094269

Sexual Minority Assessment Research Team. (2009). Best practices for asking questions about sexual orientation on surveys. Los Angeles: The Williams Institute, the University of California (UCLA) School of Law.

Sinha, S., Curtis, K., Jayakody, A., Viner, R., \& Roberts, H. (2007). 'People make assumptions about our communities': Sexual health amongst teenagers from black and minority ethnic backgrounds in East London. Ethnicity \& Health, 12, 423-441. doi:10.1080/13557850701616839

Social Exclusion Unit. (1999). Teenage pregnancy. London: The Stationery Office.

Spector, A.P., Carey, M.P., \& Steinberg, L. (1996). The sexual desire inventory: Development, factor structure, and evidence of reliability. Journal of Sex \& Marital Therapy, 22 175-190. Retrieved from http://www.tandf.co.uk/journals/titles/0092623X. asp

Stata Corporation. (2006). Stata statistical software: Release 9.2 [computer software]. College Station, TX: StataCorp LP.

Wellings, K., Field, J., Johnson, A.M., Wadsworth, J., \& Bradshaw, S. (1994). Sexual behaviour in Britain: The national survey of sexual attitudes and lifestyles. London: Penguin Books.

Wellings, K., Nanchahal, K., Macdowall, W., McManus, S., Erens, B., Mercer, C.H., . . Field, J. (2001). Sexual behaviour in Britain: Early heterosexual experience. Lancet, 358, 1843-1850. doi:10.1016/S0140-6736(01)06885-4 